\title{
Lifestyle patterns associated with diet, physical activity, body mass index and amount of recent weight loss in a sample of successful weight losers
}

Paul T Fuglestad ${ }^{1 *}$, Robert W Jeffery ${ }^{1}$ and Nancy E Sherwood ${ }^{1,2}$

\begin{abstract}
Background: Research suggests that the interaction between biological susceptibility and environmental risk is complex and that further study of behavioral typologies related to obesity and associated behaviors is important to further elucidate the nature of obesity risk and how to approach it for intervention. The current investigation aims to identify phenotypical lifestyle patterns that might begin to unify our understanding of obesity and obesity related behaviors.
\end{abstract}

Methods: Individuals who had recently lost substantial weight of their own initiative completed measures of intentional weight control behaviors and lifestyle behaviors associated with eating. These behaviors were factor analyzed and the resulting factors were examined in relation to BMI, recent weight loss, diet, and physical activity.

Results: Four meaningful lifestyle and weight control behavioral factors were identified — regularity of meals, TV related viewing and eating, intentional strategies for weight control, and eating away from home. Greater meal regularity was associated with greater recent weight loss and greater fruit and vegetable intake. Greater TV related viewing and eating was associated with greater BMI and greater fat and sugar intake. More eating away from home was related to greater fat and sugar intake, lower fruit and vegetable intake, and less physical activity. Greater use of weight control strategies was most consistently related to better weight, diet, and physical activity outcomes.

Conclusions: Compared to the individual behavior variables, the identified lifestyle patterns appeared to be more reliably related to diet, physical activity, and weight (both BMI and recent weight loss). These findings add to the growing body of literature identifying behavioral patterns related to obesity and the overall weight control strategy of eating less and exercising more. In future research it will be important to replicate these behavioral factors (over time and in other samples) and to examine how changes in these factors relate to weight loss and weight maintenance over time.

Keywords: Lifestyle patterns, Body mass index, Weight loss, Diet, Physical activity

\footnotetext{
* Correspondence: fugl0025@umn.edu

'Division of Epidemiology and Community Health, School of Public Health, University of Minnesota, 1300 S 2nd Street, suite 300, Minneapolis, MN 55454, USA

Full list of author information is available at the end of the article
} 


\section{Background}

The high profile of obesity as a health issue continues to kindle interest in better understanding the factors that are responsible for obesity susceptibility and difficulty in adhering to lifestyle treatment. Long before the current epidemic was recognized it was clearly established that obesity runs in families and, therefore, that underlying biology is an important factor [1-3]. The unfolding population epidemic, likewise, has underscored the importance of factors in the environment that have recently resulted in dramatic changes in risk over relatively short periods of time $[4,5]$. As research progresses, however, it is becoming clear that the interaction between biological susceptibility and environmental risk is not a simple one and that further study of behavioral typologies of phenotypes predicting obesity risk may be important to further elucidate the nature of risk and how to approach it for intervention [6-8]. An ever increasing list of variables at the interface of biology and environment is now being reported, mostly from cross-sectional and longitudinal studies of populations with different characteristics, e.g., general population, weight-loss study volunteers, and successful losers, and with different age and ethnic characteristics. Among the variables most consistently associated with obesity risk are lifestyle issues like where and what people eat (e.g., fast food, sugarsweetened beverages, watching TV), when they eat (e.g., meal skipping), and intentional weight management practices (e.g., self-weighing and meal planning) [9-19].

Although studies have been able to identify various practices associated with obesity risk, there is great heterogeneity in results with even relatively consistent behaviors such as intake of fast food [17]. Furthermore, many studies tend to focus on a limited number of behaviors, and even those with a broader coverage of behaviors have seldom considered the interrelations among behavioral practices. One reason for the heterogeneity in results may be that by focusing on single behavioral indicators, researchers miss the integrated general behavioral patterns that are associated with weight, diet, and physical activity.

Building on prior research suggesting that health behaviors tend to be interrelated [20-24], the current paper presents an analysis of the relationships among a diverse array of variables related to obesity and associated behaviors (i.e., diet and physical activity) with the aim of beginning to identify phenotypical lifestyle patterns that might unify our understanding of obesity, diet, and physical activity. The population under consideration is a unique one, being comprised of adults who have recently lost significant weight on their own initiative and have volunteered for a study intended to help them keep it off. The potentially phenotypical variables fall into the following broad behavioral classes: lifestyle behaviors associated with eating (e.g., eating at fast food restaurants and convenience stores, frequency of meals and snacks, and TV viewing) and intentional weight control behaviors (e.g., counting calories, planning meals and exercise to manage weight). Recent research by Sciamanna et al. [18] underscores the importance of considering the unique relations of behavioral practices to multiple aspects of obesity and weight change (e.g., weight loss and weight maintenance). In this respect, the present analysis examines the outcome variables of weight, defined by BMI at study entry, the amount of weight lost in the recent weight loss episode that qualified participants for study eligibility, and behaviors most proximal to weight regulation, namely diet and physical activity. Utilizing factor analysis, we aim to identify unifying themes of behavior and self-regulation that relate to obesity, success in self-initiated weight loss, diet, and physical activity.

\section{Methods}

\section{Participants}

Participants were recruited for this study from the general population through public advertisement in the St. Paul/Minneapolis area in the USA. The key criterion for study entry was having intentionally lost at least $10 \%$ of body weight in the past year. The total sample included 419 adults (82\% female; $87 \%$ non-Hispanic white; $64 \%$ college or graduate degree; age $M=47$; BMI $M=28$; $\%$ weight loss $M=16$ ). Consent was obtained from all participants and the University of Minnesota's Institutional Review Board Human Subjects Committee approved all protocols. For additional details of participant characteristics, recruitment, and intervention procedures see Sherwood et al. [25].

\section{Measures}

Table 1 shows descriptive statistics for all measures. Variables in the two lifestyle categories as well as the outcome variables are defined below.

\section{Lifestyle behaviors}

Participants indicated how many times during the past week they 1) ate breakfast, 2) ate lunch, 3) ate dinner, 4) ate after 7 p.m., 5) ate a snack while watching TV, 6) ate a meal while watching TV, 7) ate food at work (provided by an employer or other employee), 8) ate food prepared at a fast food restaurant, 9) ate food prepared at a sitdown restaurant, 10) purchased food at a convenience store/gas station, and 11) purchased food for a fundraiser. Response options for each question were 0,1 or 2,3 or 4, 5 or 6, and 7+. Additionally, participants indicated how many hours of TV they watch on the average weekday and weekend day using the response options $0,<1$, 1, 2, 3, 4, and 5+. After appropriate weighting, weekday 
Table 1 Baseline descriptive statistics for study variables

\begin{tabular}{|c|c|}
\hline \multicolumn{2}{|l|}{ Lifestyle Behaviors } \\
\hline Eat breakfast (daily) & $62 \%$ \\
\hline Eat lunch (daily) & $62 \%$ \\
\hline Eat dinner (daily) & $72 \%$ \\
\hline Eat after 7 p.m. $(3+/$ week $)$ & $60 \%$ \\
\hline Eat snacks with TV (3 +/week) & $37 \%$ \\
\hline Eat meals with TV (3 +/week) & $46 \%$ \\
\hline TV viewing $(2+\mathrm{hr} /$ day $)$ & $47 \%$ \\
\hline Eat food from work (1 +/week) & $43 \%$ \\
\hline Eat food from sit down (1 +/week) & $70 \%$ \\
\hline Eat fast food ( 1 +/week) & $48 \%$ \\
\hline Eat food from convenience store ( $1+/$ week) & $15 \%$ \\
\hline Eat food from fundraiser (1 +/week) & $1 \%$ \\
\hline \multicolumn{2}{|l|}{ Weight control Behaviors } \\
\hline Self-weighing (daily + ) & $47 \%$ \\
\hline Write down calorie content (sometimes + ) & $25 \%$ \\
\hline Write down exercise (sometimes + ) & $40 \%$ \\
\hline Use meal replacements (sometimes + ) & $21 \%$ \\
\hline Plan meals (often + ) & $66 \%$ \\
\hline Plan exercise $($ often + ) & $62 \%$ \\
\hline \multicolumn{2}{|l|}{ Outcome Variables } \\
\hline Body mass index & $28.5(4.9)$ \\
\hline$\%$ weight lost at baseline & $16.2(5.3)$ \\
\hline $\begin{array}{l}\text { Fruits (servings per day; excluding } \\
\text { juice and fruits in foods such as pies) }\end{array}$ & $1.6(1.2)$ \\
\hline $\begin{array}{l}\text { Vegetables (servings per day; } \\
\text { excluding starchy vegetables and } \\
\text { vegetables in foods such as stews or pot pies) }\end{array}$ & $1.1(.85)$ \\
\hline Discretionary fat (grams per day) & $44.9(27.9)$ \\
\hline Added sugars (teaspoons per day) & $11.4(9.0)$ \\
\hline Physical Activity (kcal per week) & 1998 (1688) \\
\hline
\end{tabular}

Percentages of participants performing a given behavior are shown for lifestyle and weight control behaviors. Means and standard deviations are shown for continuous outcome variables.

and weekend viewing were combined to form an index of average daily viewing.

\section{Weight control strategies}

Participants reported how often they weigh themselves using the response options never, once a year or less, every couple of months, every month, every week, every day, and more than once a day. They also reported how often they 1) write down the calorie content of the foods they eat, 2) write down the amount and type of exercise they do, 3) use meal-replacement products to manage their weight, 4) plan their meals to manage their weight, and 5) plan their exercise to manage their weight using the response options: never, rarely, sometimes, often, and very often.
$B M I$

Weight and height were measured in person with participants in light clothing without shoes (Seca 770 Medical Scale; Seca 214 Portable Height Rod). BMI $\left(\mathrm{kg} / \mathrm{m}^{2}\right)$ was computed.

\section{Weight loss}

During the initial phone screening, weight loss in the last year was computed by subtracting self-reported current body weight from self-reported highest body weight during the past year. Weight loss was also computed at baseline by subtracting measured baseline body weight from self-reported highest body weight. The later variable is used in subsequent analysis as the measure of recent weight loss. Adopting procedures from the $\mathrm{Na}$ tional Weight Control Registry (NWCR) [26], potential participants were required to document their recent loss (e.g., "before-and-after" photographs, names of individuals able to verify weight loss). This was done to increase assurance of the veracity of their self-reported weight loss. Amount of recent weight loss was unrelated to BMI $(r=-.07, p=.13)$.

\section{Diet}

Dietary intake was assessed using the National Cancer Institute's Web-based Diet History Questionnaire (DHQ). Several studies have documented the reliability and validity of the paper-and-pencil version of this measure $[27,28]$, and one advantage of the web-based DHQ is that respondents cannot complete the questionnaire with missing or inconsistent responses. The DHQ asks about the frequency of eating and drinking 124 items over the past year and includes portion size and dietary supplement questions. The data is then analyzed using software developed by $\mathrm{NCI}$ to yield nutrient intake and food group servings. Servings per day of fruits (excluding juice and fruits in other foods such as pies) and vegetables (excluding starchy vegetables and vegetables in other foods such as stews or pot pies) were combined (average of z-scores) to form an index of more healthful eating, whereas intake of discretionary fat (grams per day) and added sugars (teaspoons per day) were combined (average of $\mathrm{z}$-scores) to form an index of less healthful eating.

\section{Physical activity}

Physical activity was assessed using the Paffenbarger Physical Activity Questionnaire [29]. This instrument asks individuals to indicate the number of city blocks walked, flights of stairs climbed, and light ( $5 \mathrm{kcal} / \mathrm{min})$, medium $(7.5 \mathrm{kcal} / \mathrm{min})$, and heavy $(10 \mathrm{kcal} / \mathrm{min})$ leisure time activities in the past week. The caloric expenditure from each of these activities was summed to estimate total kcal of energy expenditure per week (beyond basal 
metabolic rate). The Paffenbarger questionnaire has been shown to have satisfactory reliability and predictive validity $[30,31]$.

\section{Analysis plan}

The main goal of the present research was to examine the relation of lifestyle patterns to diet, physical activity, BMI, and weight loss in the past year. Multiple regression was used when lifestyle variables were examined continuously, and ANOVA was used when lifestyle variables were examined categorically. Initial analysis examined the associations of individual behaviors with outcomes of interest. Factor analysis was then used to identify general lifestyle patterns. Next, the associations of these lifestyle factors with BMI, recent weight loss, diet, and physical activity were examined. All analyses were performed using SPSS. Covariates included in the analyses were sex, age, marital status (married versus not), and race (non-Hispanic white versus not). Cohen's $d$ is reported as an effect size indicator for mean comparisons.

\section{Results}

Relation of individual lifestyle and weight control behaviors to BMI, weight loss, diet, and physical activity

Analyses were done with lifestyle and weight control behaviors treated as continuous variables and as dichotomous high and low categories taking into account the response scales and reported frequencies of each behavior. The strength and direction of associations were very similar in the two analyses. We present the categorical results here because we felt that differences between categorical means would be easier to interpret than beta weights. For high frequency behaviors such as eating meals, the variables were dichotomized into $<$ daily and daily. For low frequency behaviors such as eating food from a convenience store, the variables were dichotomized into none and $1+$ times per week. For medium frequency behaviors such as eating after 7:00 p.m., the variables were dichotomized into $<3$ and $3+$ times per week. Table 2 shows the estimated means of BMI, weight loss, diet, and physical activity as a function of individual lifestyle and weight control behavior variables. As shown in Table 2, most behaviors were related to at least one outcome of interest and many were related to multiple outcomes. The behaviors most strongly related to BMI were watching television $\left[<2\right.$ hours $/$ day $M=27.9 \mathrm{~kg} / \mathrm{m}^{2}$ versus 2 or more hours/day $M=29.2 \mathrm{~kg} / \mathrm{m}^{2}, \quad F$ $(1,410)=7.46, p<.01, d=.26]$ and eating food from a convenience store [none $M=28.2 \mathrm{~kg} / \mathrm{m}^{2}$ versus 1 or more/week $M=30.1 \mathrm{~kg} / \mathrm{m}^{2}, \quad F(1,411)=8.11, p<.01$, $d=.39]$. The behaviors most strongly related to percentage of weight lost in the last year were eating fast food [none $M=17.0 \%$ versus 1 or more/week $M=15.3 \%, F$
$(1,412)=10.17, p<.01, d=.32]$ and the weight control strategies of writing down calorie content, [rarely or less $M=15.7 \%$ versus sometimes or more $M=17.6 \%, F$ $(1,412)=10.17, p<.01, d=.37$ ], using meal replacements [rarely or less $M=15.8 \%$ versus sometimes or more $M=17.4 \%, F(1,412)=6.02, p<.05, d=.30]$, and planning meals [sometimes or less $M=15.0 \%$ versus often or more $M=16.8 \%, F(1,412)=9.48, p<.01, d=.33]$. The behaviors most strongly related to fat and sugar intake were eating food from a convenience store [none $M=-0.07 \mathrm{z}$ score versus 1 or more/week $M=0.42$ z-score, $F$ $(1,412)=14.06, p<.001, d=.49$ ] or from a fundraiser [none $M=-0.03 \mathrm{z}$-score versus 1 or more/week $M=0.55$ $\mathrm{z}$-score, $F(1,412)=7.46, p<.01, d=.58]$ and planning meals [sometimes or less $M=0.20 \mathrm{z}$-score versus often or more $M=-0.10$ z-score, $F(1,412)=8.72, p<.01$, $d=.30]$. The behaviors most strongly related to fruit and vegetable intake were eating lunch [less than daily $M=-0.21 \quad \mathrm{z}$-score versus daily $M=0.13 \mathrm{z}$-score, $F$ $(1,412)=11.53, p=.001, d=.34$ ], eating fast food [none $M=0.17 \mathrm{z}$-score versus 1 or more/week $M=-0.17 \mathrm{z}$ score, $F(1,412)=11.42, \quad p=.001, d=.34]$, and the weight control strategies of writing down calorie content [rarely or less $M=-0.09 \mathrm{z}$-score versus sometimes or more $M=0.28$ z-score, $F(1,412)=10.66, p=.001$, $d=.37$ ] and planning meals [sometimes or less $M=$ $0.26 \mathrm{z}$-score versus often or more $M=0.14 \mathrm{z}$-score, $F$ $(1,412)=14.68, p<.001, d=.40]$. Finally, the behaviors most strongly related to physical activity were the weight control strategies of writing down exercise [rarely or less $M=1680 \mathrm{kcal}$ versus sometimes or more $M=2486 \mathrm{kcal}, F(1,412)=23.02, p<.001, d=.48]$ and planning exercise [sometimes or less $M=1236 \mathrm{kcal}$ versus often or more $M=2459 \mathrm{kcal}, F(1,412)=58.46$, $p<.001, d=.72]$.

\section{Factor analysis of lifestyle and weight control variables}

Although the mean comparisons reported in Table 2 provide some insight into individual behaviors associated with BMI, recent weight loss, diet, and physical activity, the multiple comparisons and imperfect behavioral indicators make overall interpretation difficult. Therefore, factor analysis was used to gain a clearer understanding of how the behaviors in question relate to one another and to identify general lifestyle patterns. A series of analyses using principal component analysis and promax rotation (allowing the factors to be correlated) were performed to determine the simple structure underlying the observed correlations of lifestyle and weight control behaviors (i.e., to reduce the number of variables to a smaller number of factors which account for a large amount of the variability). Based on a scree plot (there was a clear break between factors four and five) and pattern of salients (i.e., the pattern of loadings with various 
Table 2 BMI, \% recent weight lost, diet, and physical activity means as a function of lifestyle and weight control behaviors

\begin{tabular}{|c|c|c|c|c|c|c|}
\hline Lifestyle behaviors & Level & BMI & $\begin{array}{l}\% \text { weight } \\
\text { lost }\end{array}$ & $\begin{array}{l}\text { Fat \& sugar } \\
\text { (z-scores) }\end{array}$ & $\begin{array}{l}\text { Fruits \& veggies } \\
\text { (z-scores) }\end{array}$ & $\begin{array}{l}\text { Physical activity } \\
\text { (kcal per week) }\end{array}$ \\
\hline \multirow[t]{2}{*}{ Eat breakfast } & $<$ daily & 29.0 & $15.6+$ & 0.02 & $-0.15^{*}$ & $1743^{*}$ \\
\hline & daily & 28.2 & 16.5 & -0.01 & 0.10 & 2156 \\
\hline \multirow[t]{2}{*}{ Eat lunch } & $<$ daily & $29.0+$ & $15.6+$ & 0.01 & $-0.21^{* * *}$ & 1938 \\
\hline & daily & 28.1 & 16.5 & -0.01 & 0.13 & 2054 \\
\hline \multirow[t]{2}{*}{ Eat dinner } & $<$ daily & 29.1 & 16.0 & -0.01 & $-0.16^{*}$ & 2017 \\
\hline & daily & 28.3 & 16.2 & 0.01 & 0.07 & 1993 \\
\hline \multirow[t]{2}{*}{ Eat after 7 p.m. } & $<3 /$ week & $28.0+$ & $15.6+$ & -0.03 & -0.001 & 1934 \\
\hline & $3+/$ week & 28.9 & 16.6 & 0.02 & 0.01 & 2043 \\
\hline \multirow[t]{2}{*}{ Snack with TV } & $<3 /$ week & 28.4 & $15.8+$ & $-0.06+$ & -0.04 & 2095 \\
\hline & $3+/$ week & 28.7 & 16.8 & 0.11 & 0.09 & 1847 \\
\hline \multirow[t]{2}{*}{ Meal with TV } & $<3 /$ week & 28.2 & 16.3 & -0.04 & -0.07 & 2020 \\
\hline & $3+/$ week & 28.7 & 16.1 & 0.04 & 0.09 & 1987 \\
\hline \multirow[t]{2}{*}{ TV viewing } & $<2 \mathrm{hr} /$ day & $27.9^{* *}$ & $16.6+$ & $-0.07 \dagger$ & 0.06 & $2150^{*}$ \\
\hline & $2+\mathrm{hr} /$ day & 29.2 & 15.6 & 0.09 & -0.06 & 1826 \\
\hline \multirow[t]{2}{*}{ Food from work } & none & 28.6 & 16.2 & 0.01 & $0.08+$ & 1996 \\
\hline & $1+/$ week & 28.3 & 16.1 & -0.01 & -0.10 & 2005 \\
\hline \multirow[t]{2}{*}{ Food from sit down } & none & 28.4 & 16.1 & -0.06 & 0.01 & 1883 \\
\hline & 1 +/week & 28.5 & 16.2 & 0.03 & 0.004 & 2026 \\
\hline \multirow[t]{2}{*}{ Food from fast food } & none & $28.1 \dagger$ & $17.0^{* *}$ & -0.07 & $0.17^{* * *}$ & $2171^{*}$ \\
\hline & $1+/$ week & 28.9 & 15.3 & 0.08 & -0.17 & 1818 \\
\hline \multirow[t]{2}{*}{ Food from convenience store } & none & $28.2^{* *}$ & 16.3 & $-0.07^{* * *}$ & 0.02 & 2017 \\
\hline & 1 +/week & 30.1 & 15.5 & 0.42 & -0.10 & 1904 \\
\hline \multirow[t]{2}{*}{ Food from fundraiser } & none & 28.6 & 16.2 & $-0.03^{* *}$ & 0.01 & 2020 \\
\hline & $1+/$ week & 27.4 & 15.4 & 0.55 & -0.10 & 1621 \\
\hline \multirow[t]{2}{*}{ Self-weighing } & $<$ daily & 28.7 & 16.3 & $0.09+$ & 0.001 & 1900 \\
\hline & daily or more & 28.3 & 16.0 & -0.09 & 0.01 & 2110 \\
\hline \multirow[t]{2}{*}{ Write down calorie content } & rarely or less & 28.5 & $15.7^{* * *}$ & $0.05+$ & $-0.09 * * *$ & $1893^{*}$ \\
\hline & sometimes or more & 28.3 & 17.6 & -0.14 & 0.28 & 2315 \\
\hline \multirow[t]{2}{*}{ Write down exercise } & rarely or less & 28.4 & 16.0 & 0.03 & -0.03 & $1680^{* * *}$ \\
\hline & sometimes or more & 28.6 & 16.4 & -0.04 & 0.06 & 2486 \\
\hline \multirow[t]{2}{*}{ Use meal replacements } & rarely or less & 28.4 & $15.8^{*}$ & 0.001 & -0.002 & 2044 \\
\hline & sometimes or more & 28.9 & 17.4 & 0.01 & 0.02 & 1834 \\
\hline \multirow[t]{2}{*}{ Plan meals } & sometimes or less & $29.2^{*}$ & $15.0^{* *}$ & $0.20^{* *}$ & $-0.26^{* * *}$ & $1720^{*}$ \\
\hline & often or more & 28.1 & 16.8 & -0.10 & 0.14 & 2142 \\
\hline \multirow[t]{2}{*}{ Plan exercise } & sometimes or less & 28.8 & $15.5^{*}$ & $0.11 \dagger$ & -0.08 & $1236^{* * *}$ \\
\hline & often or more & 28.3 & 16.6 & -0.06 & 0.05 & 2459 \\
\hline
\end{tabular}

All ANOVAs control for age, gender, race (white versus not), and marital status (married versus not). Significance level of mean differences: $\dagger p<.10,{ }^{*} p<.05,{ }^{* *}$ $p<.01,{ }^{* * *} p<.001$

numbers of factors specified), a 4-factor solution seemed to best account for the observed correlations (see Table 3 for factor loadings). The four factors accounted for $43 \%$ of the item variability and we labeled them as 1) regularity of meals, 2) television related eating and viewing, 3) intentional weight control strategies, and 4) eating away from home. Only one behavior, self-weighing, did not have a factor loading of at least .30 on any factor (it loaded .26 on the weight control strategies factor). The regularity of meals factor was 
Table 3 Factor loadings for lifestyle and weight control behaviors

\begin{tabular}{|c|c|c|c|c|}
\hline Item & $\begin{array}{l}\text { Regularity of } \\
\text { meals }\end{array}$ & $\begin{array}{l}\text { TV related } \\
\text { viewing \& eating }\end{array}$ & $\begin{array}{l}\text { Weight control } \\
\text { strategies }\end{array}$ & $\begin{array}{l}\text { Eating away } \\
\text { from home }\end{array}$ \\
\hline Eat lunch & .87 & & & \\
\hline Eat dinner & .81 & & & \\
\hline Eat breakfast & .70 & & & \\
\hline Eat a snack while watching TV & & .78 & & \\
\hline Eat a meal while watching TV & & .76 & & \\
\hline Average daily hours of TV viewing & & .75 & & \\
\hline Eat after 7 p.m. & & .52 & & \\
\hline Write down amount and type of exercise & & & .76 & \\
\hline Write down calorie content of food & & & .70 & \\
\hline Plan meals to manage weight & & & .48 & \\
\hline Plan exercise to manage weight & & & .47 & -.35 \\
\hline Use meal replacements to manage weight & & & .41 & \\
\hline \multicolumn{5}{|l|}{ Self-weighing } \\
\hline Eat food prepared at sit down restaurant & & & & .64 \\
\hline Eat food at work (provided by employer or other employee) & & & & .60 \\
\hline Purchase food at convenience store/gas station & & & & .50 \\
\hline Eat food prepared at fast food restaurant & & & & .44 \\
\hline Purchase food for a fundraiser & & & & .32 \\
\hline
\end{tabular}

Only loadings above .3 are shown.

positively related to the weight control strategies factor $(r=.25, p<.001)$ and negatively related to the eating away from home factor $(r=-.26, p<.001)$. The weight control strategies factor was negatively related to the eating away from home factor $(r=-.14, p<.01)$. The television related eating and viewing factor and the eating away from home factor were positively related $(r=.20, p<.001)$.

\section{Relation of factors to BMI, weight loss, diet, and physical activity}

Having established that the lifestyle and weight control behaviors formed coherent, meaningful factors, we examined the relation of these factors to outcomes of interest (controlling for age, gender, race, and marital status). Similar to the analyses examining individual behavioral predictors, the lifestyle factors were treated both continuously (factor scores were computed from the analysis described above using the regression method, which predicts the location of each individual on each factor) and categorically (we divided each of the factor scores into tertiles, i.e., 3 equal groups), and the strength and direction of effects were quite similar in both cases. We again present the categorical results here because we felt that differences between categorical means would be easier to interpret than beta weights.

Table 4 shows the means of BMI, recent weight loss, diet, and physical activity broken down by tertiles of the lifestyle factor scores for regularity of meals, television related eating and viewing, weight control strategies, and eating away from home. Eating more regular meals was related to greater weight loss in the last year [lower $M=15.9 \%$ and middle $M=15.4 \%$ versus upper $M=17.3 \%$, $\mathrm{F}(2,402)=4.72, p<.05, d s=.26, .34]$ and greater fruit and vegetable intake [lower $M=-0.22 \mathrm{z}$-score versus middle $M=0.13 \quad z$-score and upper $M=0.13$ z-score, $F$ $\left.(2,402)=5.59, p<.01, d^{\prime} s=.35, .35\right]$. More use of weight control strategies was associated with a lower BMI [lower $M=29.2 \mathrm{~kg} / \mathrm{m}^{2}$ versus middle $M=27.6 \mathrm{~kg} / \mathrm{m}^{2}, \mathrm{~F}$ $(2,401)=3.61, p<.01, d=.32]$, greater weight loss in the last year [lower $M=14.9 \%$ versus middle $M=16.7 \%$ and upper $M=17.0 \%, \mathrm{~F}(2,402)=6.63, p=.001, d s=.35, .41]$, lower fat and sugar intake [lower $M=0.20 \mathrm{z}$-score versus middle $M=-0.11 \mathrm{z}$-score and upper $M=-0.08 \mathrm{z}$-score, $\mathrm{F}$ $(2,402)=4.47, p<.05, d s=.31, .28]$, greater fruit and vegetable intake [lower $M=-0.18 \mathrm{z}$-score versus upper $M=0.20 \quad$ z-score, $\mathrm{F}(2,402)=5.07, p<.01, d=.38]$, and greater physical activity [lower $M=1411 \mathrm{kcal}$ versus middle $M=1964 \mathrm{kcal}$ versus upper $M=2612, \quad \mathrm{~F}$ $(2,402)=18.87, p<.001, d s=.33$ (lower versus middle), .38 (middle versus upper), .71 (lower versus upper)]. Conversely, greater television related viewing and eating was related to greater BMI [lower $M=27.8 \mathrm{~kg} / \mathrm{m}^{2}$ and middle $M=28.0 \mathrm{~kg} / \mathrm{m}^{2}$ versus upper $M=29.5, \quad \mathrm{~F}$ $(2,401)=5.11, p<.01, d s=.35, .31]$ and higher fat and sugar intake [lower $M=-0.22 \quad \mathrm{z}$-score versus middle 


\begin{tabular}{|c|c|c|c|c|c|c|}
\hline Factor & Tertile & BMI & $\%$ weight lost & $\begin{array}{l}\text { Fat \& sugar } \\
\text { (z-scores) }\end{array}$ & $\begin{array}{l}\text { Fruits \& veggies } \\
\text { (z-scores) }\end{array}$ & $\begin{array}{l}\text { Physical activity } \\
\text { (kcal per week) }\end{array}$ \\
\hline \multirow[t]{3}{*}{ Regularity of meals } & Lower & 29.0 & $15.9 \mathrm{a}$ & 0.04 & $-0.22_{a}$ & 1962 \\
\hline & Middle & $28.6+$ & $15.5_{a}^{*}$ & 0.06 & $0.13_{b}^{* *}$ & $1777+$ \\
\hline & Upper & 27.7 & $17.3_{b}$ & -0.09 & $0.13_{b}$ & 2253 \\
\hline \multirow[t]{3}{*}{ TV related eating and viewing } & Lower & $27.8 \mathrm{a}$ & 16.4 & $-0.22_{a}$ & -0.05 & 2168 \\
\hline & Middle & $28.0_{a}^{* *}$ & 16.0 & $0.09_{b}^{* *}$ & 0.04 & 2011 \\
\hline & Upper & $29.5 b$ & 16.3 & $0.14_{b}$ & 0.04 & 1813 \\
\hline \multirow[t]{3}{*}{ Weight control strategies } & Lower & $29.2_{a}$ & $14.9 \mathrm{a}$ & $0.20_{a}$ & $-0.18 \mathrm{a}$ & $1411_{a}$ \\
\hline & Middle & $27.6_{b}^{*}$ & $16.7^{* * *}$ & $-0.11_{b}^{*}$ & $0.02_{a, b}{ }^{* *}$ & $196 b_{b}^{* * *}$ \\
\hline & Upper & $28.5_{a, b}$ & $17.0_{\mathrm{b}}$ & $-0.08_{b}$ & $0.20_{b}$ & $2612_{c}$ \\
\hline \multirow[t]{3}{*}{ Eating away from home } & Lower & 27.8 & 16.7 & $-0.16_{a}$ & $0.11_{a}$ & $2294 a$ \\
\hline & Middle & 28.7 & 16.4 & $-0.02_{a, b} b^{* *}$ & $0.08_{a}^{*}$ & $2106_{a}^{* * *}$ \\
\hline & Upper & 28.8 & 15.6 & $0.19_{b}$ & $-0.16_{b}$ & $1586_{b}$ \\
\hline
\end{tabular}

All ANOVAs control for age, gender, race (white versus not), and marital status (married versus not). Significance level for overall ANOVAs: $\dagger<.10,{ }^{*} p<.05$, ${ }^{* *} p<.01,{ }^{* * *} p<.001$. Means with different subscripts differ at $p<.05$.

$M=0.09 \quad$ z-score and upper $M=0.14 \quad$ z-score, $\quad F$ $(2,402)=5.90, p<.01, d s=.31, .36]$. More eating away from home was related to greater fat and sugar intake [lower $M=-0.16 \mathrm{z}$-score versus upper $M=0.19 \mathrm{z}$-score, $\mathrm{F}(2,402)=4.77, p<.01, d=.35]$, lower fruit and vegetable intake [lower $M=0.11 \mathrm{z}$-score and middle $M=0.08 \mathrm{z}$ score versus upper $M=-0.16 \quad z$-score, $\mathrm{F}(2,402)=3.07$, $p<.05, d s=.27, .24$ ], and less physical activity [lower $M=2294 \mathrm{kcal}$ and middle $M=2106 \mathrm{kcal}$ versus upper $M=1586 \mathrm{kcal}, \mathrm{F}(2,402)=6.70, p=.001, d s=.42, .31]$.

\section{Discussion}

The analyses presented in this paper used factor analysis to combine variables that have previously been found to be associated with body weight and risk of weight gain [9-19]. Twelve questions about regularity and location of eating as well as TV viewing and six questions about intentional strategies used for weight control were factor analyzed. A four-factor solution seemed to best fit the data, which we labeled regularity of meals, TV viewing and eating, intentional strategies for weight control, and eating away from home. These factors were examined in relation to degree of success in a recent weight control attempt, food intake, physical activity, and current BMI. These analyses clearly showed that variables with heuristic similarity tend to group together, e.g., individuals who eat breakfast regularly also tend to eat other meals more regularly. Qualitatively, it appears that combining these variables into meaningful groupings produced variables that were related to behavioral and weight outcomes more consistently than the individual variables from which they were derived. For example, the weight control factor was consistently related to all weight, diet, and physical activity variables, whereas the individual weight control behaviors varied substantially in terms of significant associations with weight, diet, and physical activity variables. Additionally, whereas none of the individual meals variables were significantly related to recent weight loss, the regularity of meals factor was significantly related to recent weight loss.

Most of the observed associations with outcomes were in the direction that would be expected, as were their associations with each other. There were a few surprises, however. For example, TV viewing and eating was strongly associated with BMI and fat and sugar intake, but not with success in recent weight control efforts or fruit and vegetable intake and physical activity. Eating regular meals was associated with greater success in recent weight control efforts and higher fruit and vegetable intake, but was not associated with fat and sugar intake and only weakly related to BMI and physical activity. Greater eating away from home was associated with less healthful eating and physical activity behaviors, but was not significantly related to BMI or recent weight loss (although the trends are in the expected direction). Intentional weight control strategies were most consistently related to healthier behaviors, healthier BMI, and greater success in recent weight control efforts. The latter finding is clearly supportive of intentional weight control efforts as healthy behavior. However, all participants in this study were required to have lost $10 \%$ or more of their body weight within the last year, which excluded any individuals who tried to lose weight but without any success.

Including only successful losers in this sample poses clear problems with generalizing from these results to 
the general population. The sample is also fairly high in SES and weighted toward women. While the sample has limitations, we believe it also offers a unique insight into the processes of self-initiated weight loss and suggests that even among those who try to lose weight and are successful there is still a dose response relationship between degree of weight control effort and recent weight loss and BMI. Unexpectedly, self-weighing did not load highly onto the weight control factor and was not related to weight in this sample. However, there may have been a restriction of range issue for this behavior as most participants engaged in at least weekly weighing $(88 \%)$, and the other weight control behaviors had greater variability. In future research it will be important to examine this diverse array of lifestyle behaviors in a more representative sample.

The results of this study do not answer precisely the question about obesity risk phenotypes. However, they suggest to us some possible directions for additional research. For example, regularity of eating meals was related to greater weight loss in the past year, whereas eating out was related to poorer diet and exercise habits and TV related eating and viewing was related to greater BMI. This suggests that individuals with more ordered eating lives may have more weight control success. Is having an ordered eating life an indication of a broader mastery of self-control skills, or a better sense of proportion in making commitments? It also appears that meal regularity and weight control strategies were relatively more strongly associated with the positive outcomes of recent weight loss and eating fruits and vegetables, whereas TV related eating and viewing and eating away from home were relatively more associated with the negative outcomes of BMI and fat and sugar intake. This pattern of results suggests that the identified behavioral factors can be thought of in terms of relatively more regulated and unregulated lifestyle patterns (correlation among the factors also suggests this). Thus, an important question is the extent to which these patterns also differentially predict weight control over time? Other research suggests that disordered lifestyle patterns (e.g., disordered work schedule, disordered meal patterns) are related to weight gain over time $[16,19,32]$.

We have used factor analysis, specifically, principal component analysis, in an attempt to simplify and organize a diverse set of behaviors. Other data reduction techniques could have been used to examine the data. For example, one might wish to identify behavioral clusters based on a particular outcome of interest. In this way, one could identify groups of behaviors that discriminate between eating fruits and vegetables or not or exercising a lot or a little. One might also wish to try to cluster individuals together based on a number of different behaviors. In this way one could examine diet, physical activity, and weight for particular clusters of people. For example, Sanchez et al. [22] found that overweight and obese women were likely to have multiple lifestyle risk behaviors (e.g., not meeting dietary and physical activity guidelines) and Boone-Heinonen et al. [24] found that prevalent and incident obesity were related to behavioral clusters (based on a number of diet and physical activity behaviors) in adolescent boys and girls. Given the cross-sectional nature of our data and our focus on a diverse set of lifestyle behaviors, factor analysis was an appropriate method for identifying unifying themes.

Recognizing that obesity is a multifaceted problem, it may also be important to link these behavioral patterns to environmental (e.g., availability of convenience foods, structural features of neighborhoods) and psychological influences (e.g., disinhibition, restraint, susceptibility to hedonically pleasing foods) that have been implicated in obesity risk [4,5,33-35]. In this way, the pattern of influences on obesity can be more fully established (e.g., environmental and psychological/biological influences $\rightarrow$ behavioral patterns $\rightarrow$ diet and physical activity $\rightarrow$ weight, weight change). For example, higher levels of disinhibition with respect to food are associated with current and prospective weight gain $[33,34]$. It may be that our TV and eating away from home factors are associated with psychological disinhibition and lead to weight gain over time.

Drawing on distinctions between behavioral initiation and maintenance $[18,36]$, we aim to establish the relation of these behavioral patterns to weight change over time. Furthermore, we plan to examine factor change over time and the relation of factor change to weight maintenance. For example, TV related eating and viewing was related to BMI, but was unrelated to the current weight loss episode. Over time will this group of behaviors predict weight gain? Furthermore, will these factors have an overall effect on weight gain? Research using large cohorts suggests that various lifestyle behaviors (e.g., specific dietary components, physical activity, and sleep) have a substantial aggregate effect on weight gain over time [37]. It is important to note that research examining aggregate effects of behavior on weight over time [37] or the differential effects of particular behaviors with weight loss and weight maintenance [18] have not examined the effects of behavioral factors (derived via factor analysis) on weight over time.

\section{Conclusions}

In a study of people who lost substantial weight of their own initiative (at least 10 percent in the past year), we were able to identify four lifestyle and weight control behavioral factors related to obesity-regularity of meals, weight control strategies, TV related eating 
and viewing, and eating away from home. Compared to the individual behavior variables, these factors appeared to be more reliably related to BMI, recent weight loss, diet, and physical activity. In general, eating away from home and TV related eating and viewing were more related to negative outcomes (BMI, fat and sugar intake), whereas eating regular meals and using weight control strategies were more related to positive outcomes (current weight loss, eating fruits and vegetables). Greater use of weight control strategies was most consistently related to better weight, diet, and physical activity outcomes. These findings add to the growing body of literature identifying behavioral patterns related to obesity risk and the overall strategy of eating less and exercising more [18,37]. In future research it will be important to replicate these behavioral factors (over time and in other samples) and to examine how changes in these factors relate to weight loss and weight maintenance over time.

\section{Competing interests}

The authors declare they have no competing interests.

\section{Authors' contributions}

NES and RWJ contributed to the conception and design of the project. PTF performed the analysis and all authors contributed to the interpretation. PTF and RJW drafted the manuscript and NES provided critical revision. All authors edited and approved the final manuscript.

\section{Acknowledgements}

This research was supported by National Cancer Institute Grant R01CA128211 and by National Institute of Diabetes and Digestive and Kidney Diseases Award T32DK083250.

\section{Author details}

${ }^{1}$ Division of Epidemiology and Community Health, School of Public Health, University of Minnesota, 1300 S 2nd Street, suite 300, Minneapolis, MN 55454, USA. ${ }^{2}$ HealthPartners Research Foundation, 8170 33rd Ave. S., Mail stop 21111R, Bloomington, MN 55425, USA.

Received: 26 October 2011 Accepted: 21 June 2012

Published: 26 June 2012

\section{References}

1. Barsh GS, Farooqi IS, O'Rahilly S: Genetics of bodyweight regulation. Nature 2000, 404:644-651.

2. Ramachandrappa S, Farooqi IS: Genetic approaches to understanding human obesity. J Clin Invest 2011, 121:2080-2086.

3. Stunkard AJ, Foch $\Pi$, Hrubec Z: A twin study of human obesity. JAMA 1986, 256:51-54

4. Giskes K, van Lenthe F, Avendano-Pabon M, Brug J: A systematic review of environmental factors and obesogenic dietary intakes among adults: are we getting closer to understanding obesogenic environments? Obes Rev 2011, 12:e95-e106.

5. Jeffery RW, Utter J: The changing environment and population obesity in the United States. Obes Res 2003, 11(Suppl):12S-22S.

6. Crawford D, Ball K: Behavioural determinants of the obesity epidemic. Asia Pac J Clin Nutr 2002, 11(Suppl 8):S718-S721.

7. Hetherington MM, Cecil JE: Gene-environment interactions in obesity. Forum Nutr 2010, 63:195-203.

8. Hill JO: Understanding and addressing the epidemic of obesity: an energy balance perspective. Endocr Rev 2006, 27:750-761.

9. Berg C, Lappas G, Wolk A, Strandhagen E, Torén K, Rosengren A, Thelle D, Lissner L: Eating patterns and portion size associated with obesity in a Swedish population. Appetite 2009, 52:21-26.
10. Bes-Rastrollo M, Basterra-Gortari FJ, Sánchez-Villegas A, Marti A, Martínez JA, Martínez-González MA: A prospective study of eating away-from-home meals and weight gain in a Mediterranean population: the SUN (Seguimiento Universidad de Navarra) cohort. Public Health Nutr 2010, 13:1356-1363.

11. Cleland VJ, Schmidt MD, Dwyer T, Venn AJ: Television viewing and abdominal obesity in young adults: Is the association mediated by food and beverage consumption during viewing time or reduced leisure-time physical activity? Am J Clin Nutr 2008, 87:1148-1155.

12. French SA, Jeffery RW, Murray D: Is dieting good for you? Prevalence, duration and associated weight and behavior changes for specific weight loss strategies over four years in US adults. Int J Obes 1999, 23:320-327.

13. Linde JA, Jeffery RW, French SA, Pronk NP, Boyle RG: Self-weighing in weight gain prevention and weight loss trials. Ann Behav Med 2005, 30:210-216.

14. Malik VS, Schulze MB, Hu FB: Intake of sugar-sweetened beverages and weight gain: a systematic review. Am J Clin Nutr 2006, 84:274288.

15. McGuire MT, Wing RR, Klem ML, Hill JO: Behavioral strategies of individuals who have maintained long-term weight losses. Obes Res 1999, 7:334-341.

16. Raynor HA, Jeffery RW, Ruggiero AM, Clark JM, Delahanty LM, Look AHEAD (Action for Health in Diabetes) Research Group: Weight loss strategies associated with BMI in overweight adults with type 2 diabetes at entry into the Look AHEAD (Action for Health in Diabetes) trial. Diabetes Care 2008, 31:1299-1304.

17. Rosenheck R: Fast food consumption and increased caloric intake: a systematic review of a trajectory towards weight gain and obesity risk. Obes Rev 2008, 9:535-547.

18. Sciamanna CN, Kiernan M, Rolls BJ, Boan J, Stuckey H, Kephart D, Miller CK, Jensen G, Hartmann TJ, Loken E, Hwang KO, Williams RJ, Clark MA, Schubart JR, Nezu AM, Lehman E, Dellasega C: Practices associated with weight loss versus weight-loss maintenance results of a national survey. Am J Prev Med 2011, 41:159-166.

19. Timlin MT, Pereira MA: Breakfast frequency and quality in the etiology of adult obesity and chronic diseases. Nutr Rev 2007, 65:268-281.

20. French SA, Hennrikus DJ, Jeffery RW: Smoking status, dietary intake, and physical activity in a sample of working adults. Health Psychol 1996, 15:448-454.

21. Kvaavik E, Andersen LF, Klepp Kl: The stability of soft drinks intake from adolescence to adult age and the association between long-term consumption of soft drinks and lifestyle factors and body weight. Public Health Nutr 2005, 8:149-157.

22. Sanchez A, Norman GJ, Sallis JF, Calfas KJ, Rock C, Patrick K: Patterns and correlates of multiple risk behaviors in overweight women. Prev Med 2008, 46:196-202.

23. Schuit AJ, van Loon AJ, Tijhuis M, Ocke M: Clustering of lifestyle risk factors in a general adult population. Prev Med 2002, 35:219-224.

24. Boone-Heinonen J, Gordon-Larsen P, Adair LS: Obesogenic Clusters: multidimensional adolescent obesity-related behaviors in the U.S. Ann Behav Med 2008, 36:217-230.

25. Sherwood NE, Crain AL, Martinson BC, Hayes MG, Anderson JD, Clausen JM, O'Connor PJ, Jeffery RW: Keep it off: a phone-based intervention for longterm weight-loss maintenance. Contemp Clin Trials 2011, 32:551-560.

26. Klem ML, Wing RR, McGuire MT, Seagle HM, Hill JO: A descriptive study of individuals successful at long-term maintenance of substantial weight loss. Am J Clin Nutr 1997, 66:239-246.

27. Thompson FE, Subar AF, Brown CC, Smith AF, Sharbaugh CO, Jobe JB, Mitt $B$, Gibson JT, Ziegler RG: Cognitive research enhances accuracy of food frequency questionnaire reports: results of an experimental validation study. J Am Diet Assoc 2002, 102:212-225.

28. Subar AF, Thompson FE, Kipnis V, Midthune D, Hurwitz P, McNutt S, McIntosh A, Rosenfeld S: Comparative validation of the Block, Willett, and National Cancer Institute food frequency questionnaires: the Eating at America's Table Study. Am J Epidemiol 2001, 154:1089-1099. 
29. Paffenbarger RS Jr, Wing AL, Hyde RT: Physical activity as an index of heart attack risk in college alumni. Am J Epidemiol 1978, 108:161-175.

30. Pereira MA, FitzerGerald SJ, Gregg EW, Joswiak ML, Ryan WJ, Suminski RR, Utter AC, Zmuda JM: A collection of physical activity questionnaires for health-related research. Med Sci Sports Exerc 1997, 29:S1-S205.

31. Harris J, French S, Jeffery R, McGovern P, Wing R: Dietary and physical activity correlates of long-term weight loss. Obes Res 1994, 2:307-313.

32. Miller DP, Han WJ: Maternal nonstandard work schedules and adolescent overweight. Am J Public Health 2008, 98:1495-1502.

33. Hays NP, Bathalon GP, McCrory MA, Roubenoff R, Lipman R, Roberts SB: Eating behavior correlates of adult weight gain and obesity in healthy women aged 55-65 y. Am J Clin Nutr 2002, 75:476-483.

34. Savage JS, Hoffman L, Birch LL: Dieting, restraint, and disinhibition predict women's weight change over 6 y. Am J Clin Nutr 2009, 90:33-40.

35. Lowe MR, Butryn ML, Didie ER, Annunziato RA, Thomas JG, Crerand CE, Ochner CN, Coletta MC, Bellace D, Wallaert M, Halford J: The Power of Food Scale. A new measure of the psychological influence of the food environment. Appetite 2009, 53:114-118.

36. Rothman AJ, Baldwin AS, Hertel AW, Fuglestad PT: Self-regulation and behavior change: Disentangling behavioral initiation and behavioral maintenance. In Handbook of self-regulation: Research, theory, and applications. 2nd edition. Edited by Baumeister RF, Vohs KD. New York: Guilford Press; 2011:574-591.

37. Mozaffarian D, Hao T, Rimm EB, Willett WC, Hu FB: Changes in diet and lifestyle and long-term weight gain in women and men. $N$ Engl J Med 2011, 364:2392-2404.

doi:10.1186/1479-5868-9-79

Cite this article as: Fuglestad et al:: Lifestyle patterns associated with diet, physical activity, body mass index and amount of recent weight loss in a sample of successful weight losers. International Journal of Behavioral Nutrition and Physical Activity 2012 9:79.

\section{Submit your next manuscript to BioMed Central and take full advantage of:}

- Convenient online submission

- Thorough peer review

- No space constraints or color figure charges

- Immediate publication on acceptance

- Inclusion in PubMed, CAS, Scopus and Google Scholar

- Research which is freely available for redistribution 